\title{
Innovación docente: los alumnos diseñan un manual cooperativo mediante el flipclass
}

\author{
Victoria Esgueva López ${ }^{\mathrm{a}}, \mathbf{M}^{\mathrm{a}}$ Angeles Carabal Montagud \\ ${ }^{a}$ Departamento de Dibujo, Universitat Politècnica de València, maeslo2@dib.upv.es. ${ }^{\text {b Departamento }}$ \\ de Conservación y Restautración de Bienes Culturales. Universitat Politècnica de València,
}

\begin{abstract}
This teaching innovation allows students to generate a theoretical-practical document, which includes all the graphic techniques used in the field of drawing, putting into practice the flipclass method. A system that reverses the traditional teaching process, so it starts working at home and ends with an autonomous and group work in the classroom.

Innovation takes advantage of the synergy that fosters collaborative work. Because the efforts of each group are combined, providing a fragment of knowledge, achieving maximum results to be shared by all students. On the other hand, it facilitates teachers to evaluate transversal competences such as team work and leadership, or effective communication.
\end{abstract}

Keywords: flipclass, cooperative work, transversal skills, drawing techniques, theoretical-practical analysis.

\begin{abstract}
Resumen
Esta innovación docente permite al alumnado generar un documento teóricopráctico, que recoge todas las técnicas gráficas usadas en el ámbito del dibujo, poniendo en práctica el método de flipclass. Un sistema que invierte el proceso de enseñanza tradicional, por lo que empieza trabajando en casa y acaba con un trabajo autónomo y grupal en el aula.

La innovación aprovecha la sinergia que propicia el trabajo colaborativo. Porque se combinan los esfuerzos de cada grupo, que aporta un fragmento de conocimiento, consiguiendo maximizar los resultados al ser compartidos por todos los alumnos. Por otra parte facilita al profesorado la evaluación de competencias transversales como el trabajo en equipo y liderazgo, o la comunicación efectiva.
\end{abstract}

Palabras clave: flipclass, trabajo cooperativo, competencias transversales, técnicas de dibujo, análisis teorico-práctico. 


\section{Introducción}

La asignatura de Procesos, técnicas y materiales del Dibujo es una asignatura fundamentalmente práctica, basada en el trabajo de taller. En este tipo de disciplinas la parte teórica, si se imparte de manera pasiva a modo de clase tradicional, resulta tediosa para el alumno y por otra parte, dificulta su evaluación al no haber en la guía docente actos de evaluación específicos de pruebas de respuestas abiertas.

Por eso se ha diseñado un método activo que obligue al alumnado a trabajar implicándose en su propio aprendizaje, que requiera su participación más dinámica y constante. Para ello nos centramos en dos conceptos que ayudan a poner en práctica este fin, es la clase inversa y el trabajo colaborativo.

La clase inversa o flipped classroom, es un método que exige al alumno mayor constancia y le obliga implicarse y ser autónomo en su aprendizaje.

"De esta forma, se hace un uso más efectivo del tiempo en el aula, dedicándolo a actividades de aprendizaje centradas en los estudiantes. En realidad, los estudiantes no necesitan tanto al profesor en el aula, durante la exposición teórica, sino más bien en la resolución de problemas.“ (BOIGUESA, F.J., ESTRUCHB, V. y VIDAL, A. 2017).

Por lo tanto, es un sistema que obliga al alumno a interaccionar entre sus iguales y con el profesor. Además exige un seguimiento más sistemático por parte del profesor, que se traduce en la creación de unas pautas de trabajo continuadas para el alumnado.

De la misma manera, la competencia transversal trabajo en equipo y liderazgo, es fundamental para el desarrollo profesional del alumno.

"En la vida profesional la competencia de trabajo en equipo es requerida en dos de cada tres demandas de puestos de trabajo con cierto nivel de responsabilidad. Se pide capacidad para integrarse en un equipo de trabajo, dotes para crear y dirigir equipos, etc. De hecho, los procesos de selección dedican parte de la entrevista a profundizar en este tema, cuando no incluyen en los mismos procesos entrevistas de grupo o actividades llamadas dinámicas de grupo, si bien tan sólo tratan de observar el desempeño de los asistentes cuando se hallan en un contexto grupal”. (VILLA, A. y POBLETE, M. 2007)

En el diseño de la presente innovación se ha tenido en cuenta que sea un tarea atractiva, para ello se solicita la participación grupal. Y la finalidad es generar un manual teóricopráctico de las técnicas del dibujo, que pasará a ser propiedad colectiva. Se plantea que cada grupo hace una parte y luego todos los grupos comparten esa información, teniendo un documento específico primordial para cursos posteriores.

Además se han unido estos planteamientos, para facilitar que los alumnos establezcan vínculos entre ellos y pautas de trabajo autónomo. Que afiancen los conceptos clave y obtengan un aprendizaje más profundo. Y a su vez, este diseño es una herramienta que posibilita al profesorado la evaluación de la parte teórico-práctica en los contenidos genéricos de la asignatura, así como las competencias transversales.

(cc) EY-NC-ND 2018, Universitat Politècnica de València

Congreso IN-RED (2018) 


\section{Objetivos}

Los objetivos que afronta este ejercicio de innovación se estructuran desde dos puntos de vista: por una parte los que hacen referencia al alumnado y por otra los que atañen al profesor.

Los objetivos que atienden a la enseñanza del alumnado, (aprendizaje):

1. Motivar e implicar al alumnado.

2. Conseguir un trabajo en equipo.

3. Conocer el sistema de enseñanza basado en la clase inversa.

4. Saber hacer una exposición formal y publica de su trabajo.

5. Realizar un manual de técnicas gráficas que recoja la parte teórica y práctica en el uso de procedimientos del dibujo. y que quede para ellos como material técnico para su uso posterior.

Y los objetivos que hacen referencia al profesor, (didácticos):

1. Conseguir crear una acción que ayude a evaluar la parte teórica de la asignatura, sin necesidad de una prueba escrita.

2. Facilitar la evaluación de la competencia transversal CT-06 Trabajo en equipo y liderazgo en el primer nivel de dominio. Al ser una asignatura de segundo curso y punto de control de esta competencia.

3. Abordar de manera incipiente otras competencias transversales como CT-03 Análisis y resolución de problemas. CT-08 Comunicación efectiva. Planificación y CT- 012 Gestión del tiempo, porque de manera tangencial se tienen en cuenta.

4. Verificar el aprendizaje individual y colectivo.

\section{Desarrollo}

A modo de preámbulo cabe aclarar que la innovación se ha aplicado en los cursos 2015-2016 y 2016-2017 en la asignatura de Procesos, Técnicas y Materiales del Dibujo, de segundo curso del Grado de Conservación y Restauración de Bienes Culturales. Asignatura troncal de 15 créditos. Se pone en práctica en dos grupos, cada uno de 32 alumnos aproximadamente. En ambos se crean 8 grupos de entre cuatro y cinco personas, y en cada uno de ellos los alumnos se reparten las funciones.

El número de horas dedicadas a la actividad es de 7 presenciales y 8 no presenciales. Esto supone una dedicación de 1,5 créditos dentro de la asignatura.

Ante todo, es fundamental exponer al alumnado el procedimiento del trabajo mediante un enunciado detallado que indique las fases y temporalización. Porque es complejo y precisa entender la secuencialidad, al tener una parte teórica y otra práctica.

La innovación en primer lugar, ayuda a entender la vertiente teórica de las técnicas y procedimientos del dibujo que abordábamos sobretodo de forma práctica en clase. En segundo lugar quisimos que este proyecto sirviera para incorporar la competencia

(cc) EY-Nc-ND 2018, Universitat Politècnica de València

Congreso In-Red (2018) 
transversal CT-O6, Trabajo en equipo, que se debía evaluar como punto de control en la asignatura. Y por último requeríamos la utilización de un método que ayudara a dinamizar las clases teóricas. Por lo que decidimos diseñar un proyecto que mezclara estos aspectos, bajo el método de clase inversa.

\section{Parte Teórica:}

En el desarrollo del trabajo comenzamos abordando los aspectos teóricos. A partir de los videos de Facebook de la asignatura de dibujo, y de otros materiales que se facilitan, como videos, artículos, visitas físicas y virtuales a colecciones y museos, y bibliografía, los alumnos arrancan el trabajo en casa supervisando el material dado.

Se continúa con sesiones de trabajo teórico-práctico en el aula, generando foros y debates por grupos, con el fin de cumplimentar una plantilla teórica dada. Cabe decir que alguna información sí se encuentra en el material facilitado, pero otra parte exige que el grupo extraiga conclusiones propias, este proceso se realiza en presencia de la profesora que orienta los foros de debate.

Esto tiene también una ventaja para el alumnado, que se pueda evaluar una parte teórica sin la necesidad de someterse a pruebas escritas. No tendrá una prueba de respuesta abierta, aunque sí se evaluará a partir de las fichas que han de rellenar. La parte teórica complementa el trabajo de taller.

\section{Parte Práctica:}

La segunda parte, en la que entra el apartado practico, se fundamenta en las conclusiones teóricas extraídas previamente, y de nuevo, mediante la supervisión previa de material digital y elección de obras artísticas relevantes trabajan fuera del aula.

Se exige que busquen obras realizadas con esa técnica, que identifiquen el procedimiento y modo de utilización. Luego en una sesión de clase, ya en el taller, realizan un catálogo o muestrario con esas tipologías, incluyendo una ficha técnica con el procedimiento.

\section{Parte final:}

Exposición pública del proyecto. Cada grupo y alumno tiene un tiempo estipulado para desarrollar una parte del guion que han decidido y todos presentan sus trabajos con una comunicación oral utilizando programas como Power point o Prezi, a partir de un índice dado. Al acabar cada exposición pública, se genera un debate y el resto de grupos hace una valoración entre iguales. (Tabla 1).

(c) EY-NC-ND 2018, Universitat Politècnica de València 
La propuesta resultante exige que con unos ítems comunes se genere un documento o manual en el que se contemplen todas las técnicas gráficas, realizadas mediante el trabajo colaborativo. Lo que supone el reparto de tareas y roles, por tanto implica que los alumnos acepten y cumplan de las reglas del juego.

Por otra parte el profesorado evalúa dos aspectos, primero los contenidos genéricos de la asignatura con otros procesos más dinámicos, que evitan la prueba escrita, y en segundo lugar las competencias transversales por medio de rubricas institucionales, en el primer nivel de dominio, al ser una asignatura de segundo curso. (UPV, 2007).

Tabla 1. Guion desarrollado por los alumnos

\begin{tabular}{|c|}
\hline Guion que el alumno desarrolla \\
\hline $\begin{array}{l}\text { 1. VÍDEOS, MUSEOS, COLECCIONES, DOCUMENTALES, } \\
\text { BIBLIOGRAFÍA. }\end{array}$ \\
\hline $\begin{array}{l}\text { 2. PLANTILLA TÉCNICAS GRÁFICAS } \\
\text { 2.1. Técnicas húmedas, secas y mixtas }\end{array}$ \\
\hline 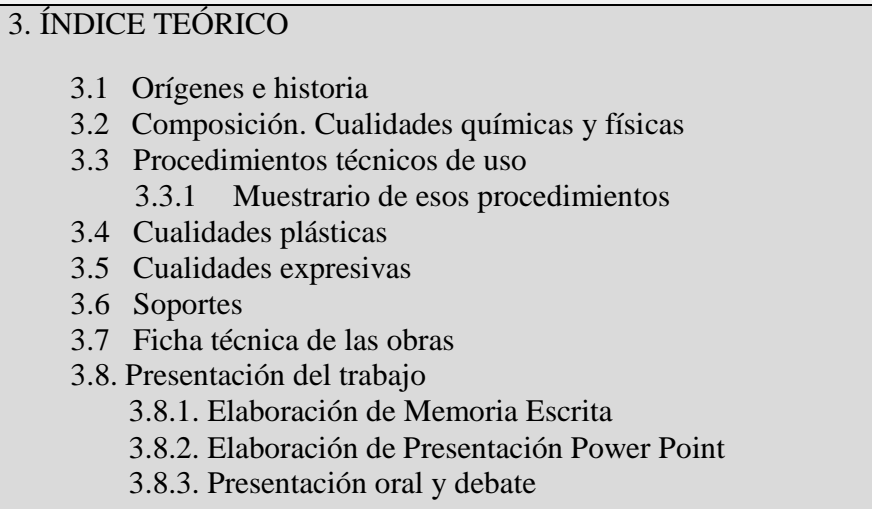 \\
\hline 3.8.4. Evaluación entre iguales \\
\hline
\end{tabular}

\section{Resultados}

Se recogen los resultados, algunos salen de evidencias objetivas como participación en el aula, notas y encuestas, mientras que otros son más interpretativos, como por ejemplo hemos notado un aumento de motivación a nivel individual. Aunque ese rasgo no se ha verificado de forma objetiva, sí lo hemos podemos observar.

La evidencias que se ven de manera clara , es la participación en el aula, todos excepto un mínimo porcentaje, alrededor de 3 \% han participado, han integrado los grupos de trabajo y han expuesto en público (Fig. 1)

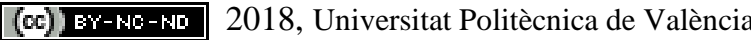




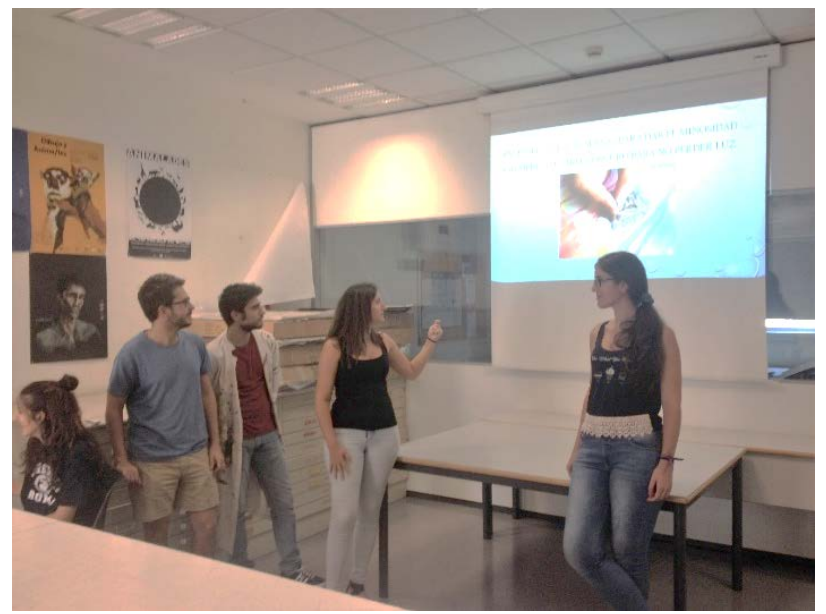

Fig. 1. Estudiantes de la asignatura Procesos, técnicas y materiales del Dibujo exponiendo.

Además se pasó una encuesta al final del curso 2015/2016, haciendoles preguntas específicas sobre la actividad.

1. ¿ ¿Te ha parecido dinámica esta actividad?

2. ¿Te ha resultado monótona?

3. ¿Crees que has aprendido más con este método grupal?

4. ¿ ¿Te ha hecho ser más exigente con los resultados?

5. ¿Prefieres la evaluación práctica por equipo?

6. ¿Prefieres la evaluacion mediante examen?

7. ¿Lo recomendarías?

8. ¿Harías algún cambio?

Los resultados de estas encuestas se recogen en la siguiente tabla. (Tabla 2)

Tabla 2. Resultados encuesta

\begin{tabular}{|c|c|}
\hline Ítems & Porcentaje \\
\hline $\begin{array}{l}\text { Dinamismo en la clase, No } \\
\text { monotonía. }\end{array}$ & $70 \%$ \\
\hline Crees que aprendes más & $20 \%$ \\
\hline Mejora nivel de autoexigencia & $66 \%$ \\
\hline $\begin{array}{l}\text { Qué mantendría, qué } \\
\text { cambiaría. }\end{array}$ & 75\% quitaría la exposion oral \\
\hline Mejor que prueba de examen & $68 \%$ \\
\hline Lo recomendarías. & $40 \%$ \\
\hline
\end{tabular}

(cc) EY-NC-ND 2018, Universitat Politècnica de València

Congreso IN-RED (2018) 
La tabla demuestra que están incomodos con la puesta en común oral, pero consideran buena opción utilizar el método de plantilla para evaluar el contenido teórico, como alternativa a la prueba escrita.

También han reconocido que el trabajo en equipo ayuda a la consecución de objetivos comunes y a la resolución de problemas porque amplía la creatividad. Además a nivel grupal, ha habido una necesidad de gestionar el tiempo y organizar encuentros del grupo fuera del aula. (Fig. 2)



Fig. 2 .Parte del estudio de técnicas .

Por otra parte, una prueba clara son las calificaciones que recoge la tabla 3, que muestra los resultados de esta parte de contenido impartida mediante el proyecto de innovación Flipclass, o anteriormente con el sistema tradicional. Se verifica con una subida consustancial.

Tabla 3. Resultados academicos

\begin{tabular}{llc}
\hline Años & Tipos de docencia & $\begin{array}{c}\text { Calificación media de } \\
\text { los dos grupos }\end{array}$ \\
\hline $2014 / 2015$ & Docencia tradicional & 6.0 \\
$2015 / 2016$, & Docencia Inversa & 6.8 \\
$2016 / 2017$ & Docencia Inversa & 6.5 \\
\hline
\end{tabular}

Destacamos para finalizar que, inicialmente se muestran reticentes al hacer una evaluación entre iguales, aunque posteriormente aumenta el debate en el aula. Además con esta práctica les ayuda a adquirir criterios definidos para valorar un proyecto teórico-práctico, tan necesarios en las titulaciones artísticas.

\section{Conclusiones}

(c) EY-NC-ND 2018, Universitat Politècnica de València

Congreso In-Red (2018) 
Esta práctica nos ha demostrado que el trabajo colaborativo y la clase inversa es un proceso que ayuda a los alumnos a implicarse en la asignatura, siendo lo más significativo que prácticamente desaparece el índice de abandono en esa actividad. ya que todos lo hacen por deferencia a sus compañeros.

También se evidencia una mejora en la actitud frente a un tema teórico, porque dinamiza más la clase y lo convierten en un foro participativo con el fin de resolver problemas. No están preocupados por estudiar sistemáticamente para la prueba escrita, porque se evalúa de otra manera. Al estar enfocada la actividad en grupos de trabajo de iguales se crea un clima en el aula más relajado, y se consigue un tipo de docencia más personalizada.

Cabe destacar, que mejora algo el rendimiento académico, se ve en el gráfico de notas. Consideramos que han conseguido un aprendizaje autónomo, con mayor implicación, y sin duda más en profundidad que se mantiene a largo plazo. Esta conclusión se puede justificar porque el tiempo empleado cuando se plantea la docencia inversa es mayor, y la actividad se estructura en plazos cortos para conseguir objetivos concretos. Ante esta exigencia, los alumnos responden con un obligado y continuado aprendizaje. Que el profesor chequea cada vez que se alcanzan los objetivos.

Por otro lado utiliza la sinergia que propicia el trabajo colaborativo, es decir que todos recogen más resultados que los que han realizado ellos mismos. Por lo tanto ellos comprueban que se optimiza su esfuerzo, ya que cada grupo hace un fragmento, y luego comparten la totalidad de las técnicas. Por tanto se quedan para ellos un documento que les será útil en otras asignaturas posteriores.

En contrapartida, se tiene que tener en cuenta que al principio los alumnos están reticentes por considerarlo una labor muy compleja. Sobretodo el último paso de la exposición pública, ya que encuentran mucha dificultad por su inexperiencia.

Además se puede cuestionar si esta iniciativa sería viable ante un grupo con un número muy elevado de alumnos. Podemos decir que en cierta medida la innovación ha funcionado, porque el número de alumnos de cada grupo, no superior a 35 , ha posibilitado que se realizara un seguimiento y una evaluación real.

\section{Referencias}

BOIGUESA, F.J., ESTRUCHB, V. y VIDAL, A. (2017).”Luces y sombras del Flipped Teaching con estudiantes de primero de turismo", en InRed 2017 III Congreso Nacional de Innovación Educativa y Docencia en Red. Valencia: Universitat Politècnica de València. 350-360 Disponible en http://ocs.editorial.upv.es/index.php/INRED/INRED2017/paper/viewFile/6788/2634 [Consulta: 3 de marzo de 2018]

DÍAZ PADILLA R. (2007) “El dibujo del natural : en la época de la postacademia”. Madrid: Akal

HAYES, C. (1992) “Guía completa de pintura y dibujo : Técnicas y materiales”. Barcelona: Tursen, S.A. Hermann Blume.

(c) ) EY-NC-ND 2018, Universitat Politècnica de València

Congreso IN-RED (2018) 
LAGE, M.J., PLATT, G.J. y TREGLIA, M. (2000). "Inverting the classroom: A gateway to creating an inclusive learning environment” en The Journal of Economic Education, vol. 31, p. 30-43.

MAYER, Ralf. (1988) “Materiales y técnicas del arte”. Barcelona: Blume.

OLTRA GUTIÉRREZ, J. V. "Evaluación en FLIP-Teaching: una alternativa a los exámenes parciales. Evaluación continua mediante tareas y casos”. en InRed 2017 III Congreso Nacional de Innovación Educativa y Docencia en Red. Valencia: Universitat Politècnica de València. 350-360 Disponible en file:///C:/Users/Rafa/Downloads/6889-18015-1-PB\%20(1).pdf [Consulta: 3 de marzo de 2018].

RIZZO, S. [et al.]. (2015). "Flipped classroom, LCA y materiales de construcción: una experiencia didáctica para una actividad de aprendizaje cooperativa y activa”. Jornades sobre Innovació Docent en Arquitectura (JIDA). "III Workshop on Educational Innovation in Architecture (JIDA'15), Barcelona School of Architecture, 25th to 29th May 2015". Barcelona: Grup per a la Innovació i la LogísticaDocent en l'Arquitectura, 2015.<http://hdl.handle.net/2117/81700> [Consulta: 8 de marzo de 2018].

UNIVERSITAT POLITÈCNICA DE VALÈNCIA (2007) “Competencias transversales". http://www.upv.es/entidades/ICE/info/U0724624.pdf [Consulta: 20 marzo 2018].

UNIVERSITAT POLITÈCNICA DE VALÈNCIA (2010). "Trabajo en equipo y liderazgo". http://www.upv.es/contenidos/COMPTRAN/info/954879normalc.html) [Consulta: 22 marzo 2018].

VILLA, A. y POBLETE, M. (2007) “Aprendizaje basado en competencias: una propuesta para la evaluación de las competencias genéricas”. Bilbao: Ediciones Mensajero. pp. 241-248. http://biblio.upmx.mx/textos/14633.pdf [Consulta: 10 marzo 2018]. 